\title{
O CUIDADO COM O SOFREDOR PSÍQUICO INSTITUCIONALIZADO
}

\author{
Marcelo Stuart Barreto ${ }^{1}$, Fátima Büchele², Elza Berger Salema Coelho ${ }^{3}$
}

RESUMO: Relato de prática assistencial de enfermagem, desenvolvido em 2003 em um hospital psiquiátrico da grande Florianópolis. Participaram do estudo 10 pacientes. O objetivo foi: resgatar habilidades de vida diária de sofredores institucionalizados, promovendo atividades educativas e habilidades pessoais, estimulando-os a realizarem atividades cotidianas. Realizamos quatro oficinas educativas nas quais foram tratados temas como: cuidado com aparência pessoal, banho, higiene oral, arrumação das camas, organização e cuidados com pertences, ingesta da medicação, alimentação e participação em atividades recreativas. Essa experiência evidenciou que o grupo de sofredores psíquicos, quando acompanhados, cuidados com atenção e orientação adequada, realizam com maior destreza as atividades consideradas básicas no cotidiano, aquelas que perderam ao longo dos anos que permanecem internados. Mostrou também vivências repletas de significados, sugerindo uma prática de cuidado de enfermagem psiquiátrica voltada ao processo de humanização, buscando alternativas simples, mas ao mesmo tempo, repleta de subsídios práticos para um grupo de pessoas que perderam a autonomia no autocuidado pelo processo institucional.

PALAVRAS-CHAVE: Saúde mental; Enfermagem psiquiátrica; Atividades cotidianas.

\section{CARING THE INSTITUTIONALIZED PSYCHIATRIC PATIENT}

\begin{abstract}
This is a nursing care practice account, carried out at a psychiatric hospital in the metropolitan area of Florianopolis/Brazil in 2003. Ten (10) patients participated in the study. It objectified: to rescue institutionalized patients' daily skills by promoting educational and personal skills activities, encouraging them to perform daily tasks. Four (4) educational workshops were held where some themes were addressed, such as: personal appearance, bath, oral hygiene, bed-making, belongings care and organization, medication intake, feeding and participation in recreational activities. This experience evidenced that mental patients when followed up, carefully cared and guided may skillfully perform basic daily tasks that they missed after years of institutionalization. It also brought about meaningful experiences, suggesting a humanized psychiatric nursing care practice, searching for simple alternatives but full of practical devices for a group of people who lost their autonomy for self-care due to the institutional process.
\end{abstract}

KEYWORDS: Mental health; Psychiatric nursing; Daily tasks.

\section{EL CUIDADO CON EL PACIENTE PSIQUICO INSTITUCIONALIZADO}

RESUMEN: Se trata de un informe de la práctica de cuidados de enfermería, que se desarrolló entre agosto y noviembre de 2003 en un hospital psiquiátrico en Florianópolis. Los participantes del estudio fueron 10 pacientes. Se llevó a través de cuatro talleres educativos sobre temas, como cuidado personal, baño, higiene bucal, camas de almacenamiento, y la organización con sus pertenencias, la ingesta de medicamentos, alimentos y participación en actividades de esparcimiento. Nuestra experiencia demuestra que el grupo de pacientes psíquicos, cuando siguió bien cuidado, con atención y orientación ajustada, logró hacer con la mayor destreza las actividades consideradas básicas en el diario, lo que se habían perdido durante los años en que estaban hospitalizados. También reveló vivencias llenas de significados, mostrando una práctica de cuidado de enfermería psiquiátrica relacionada al proceso de humanización, buscando alternativas simples, pero, al mismo tiempo, repletas de subsídios prácticos para un grupo de personas que perdieron la autonomía en autocuidado por el proceso institucional.

PALABRAS CLAVE: Salud mental; Enfermería psiquiátrica; Actividades de la vida diaria.

\footnotetext{
${ }^{1}$ Enfermeiro. Especialista em Formação Pedagógica da área da Saúde/Enfermagem-Programa Saúde da Família-Unidade Lagoa da Conceição-Florianópolis-SC.

${ }^{2}$ Professora. Doutora. Programa de Pós-Graduação em Saúde Pública. Departamento de Saúde Pública Universidade Federal de Santa Catarina-UFSC.

${ }^{3}$ Professora. Doutora. Programa de Pós-graduação em Saúde Pública-Departamento de Saúde Pública-Área de Ciências Sociais-UFSCCentro de Ciências da Saúde.
}

Autor correspondente:

Fátima Büchele

Rua Manoel Felix Cardoso, 102 - 88085-250 - Florianópolis-SC

E-mail: buchele@mbox1.ufsc.br

Recebido: 23/04/08

Aprovado: 10/10/08 


\section{INTRODUÇÃO}

A doença mental é talvez a que mais exige solidariedade humana, desprendimento, destemor, capacidade de absorção e de produção de sentimentos de cooperação e de integração social ${ }^{(1)}$. Ela rompe com uma situação de equilíbrio da dinâmica cotidiana e desorganiza de tal forma a vida do sujeito que pode torná-lo passivo e inseguro em relação à sua capacidade de sentir. Em conseqüência disso, seu estado de ânimo, auto-estima, autocuidado e sua vida de relação, ficam alteradas e instáveis.

A Organização Mundial da Saúde aponta que os transtornos mentais de 450 milhões de pessoas ainda estão longe de receberem a relevância dada a saúde física, sobretudo nos países em desenvolvimento. Esses transtornos e os de comportamento respondem por $12 \%$ da carga mundial de doenças e as verbas orçamentárias para a saúde mental, na maioria dos países, representam menos de $1 \%$ dos gastos totais em saúde, além do que $40 \%$ dos países carecem de políticas de saúde mental ${ }^{(2)}$. Assim, atividades que promovam a distração, socialização, segurança, são importantes na vida diária e constituem elemento essencial da terapêutica psiquiátrica podendo tornar possível a reintegração do sofredor psíquico na sociedade ${ }^{(3)}$.

A reabilitação psicossocial por sua vez, é um conjunto de meios que se desenvolve para facilitar a vida de pessoas com problemas severos e persistentes em saúde mental. Proporciona oportunidades de recuperação de indivíduos e minimizam efeitos desabilitantes da cronificação desses fatores por meio do desenvolvimento de atividades educativas, de insumos familiares e comunitários. Ela também incentiva o resgate de habilidades perdidas, na perspectiva de melhorar a qualidade de vida ${ }^{(4)}$.

A partir dessa abordagem inicial, consideramos as atividades educativas como um instrumento de promoção da reabilitação do sofredor psíquico e a partir desse processo desenvolvemos uma prática assistencial de enfermagem com o objetivo de resgatar habilidades de vida diária de sofredores institucionalizados, promovendo atividades educativas e habilidades pessoais, estimulando-os a realizarem atividades cotidianas.

\section{BREVE DESCRIÇÃO DA HISTÓRIA DA PSIQUIATRIA}

A existência das doenças mentais é tão antiga como a vida, mas não a sua identificação, interpretação e os tratamentos aplicados, que ao longo da história sofre transformações para acompanhar os padrões culturais, socioeconômicos, o desenvolvimento da ciência e os avanços do conhecimento na sociedade ${ }^{(3)}$.

Na pré-história, o tratamento dos doentes mentais consistia de ritos tribais com o objetivo de alterar o comportamento do doente. Se essas medidas não tivessem sucesso, o indivíduo era abandonado para morrer de inanição ou por ataque de feras ${ }^{(4)}$. Nos fins do século XVIII, no período da Revolução Francesa, Philippe Pinel buscou um tratamento mais humano para os doentes mentais, libertando-os de correntes e substituiu por camisas de força, essa medida que para a época foi uma revolução, na atualidade nos escandaliza $^{(7)}$.

No século XIX e XX, a psiquiatria tomou novos rumos que são reconhecidos no início do século $\mathrm{XXI}^{(8)}$. Em meados do século $\mathrm{XX}$, os hospitais psiquiátricos brasileiros diminuíram significativamente o número de internações, houve um maior número de altas e, em conseqüência, a necessidade da criação de novas instituições ou dispositivos assistenciais, nos quais o doente receba o tratamento sem ter de se desvincular ou separar fisicamente do seu ambiente familiar e social. Começaram a aparecer unidades psiquiátricas hospitalares, hospitais-dia, hospitais-noite e hospitais de fim-de-semana, e a política de reestruturação da assistência psiquiátrica ganhou força.

Atualmente novas modalidades terapêuticas estão sendo criadas gradativamente em nosso país. Estão sendo ampliados os atendimentos nas unidades básicas de saúde, cresce em todo Brasil a os Centros de Atenção Psicossocial (CAPS), as unidades de internação psiquiátrica em hospitais gerais e as equipes de matriciamento em saúde mental. Porém, muitos sofredores psíquicos ainda permanecem internados e cronificados nas instituições psiquiátricas. Esse processo gera a institucionalização nessas pessoas e resulta numa assistência psiquiátrica que produz a perda da dimensão temporal e favorece estados patológicos ${ }^{(9)}$.

\section{Reabilitação Psicossocial}

No Brasil, nos últimos 20 anos, vem aumentado o interesse em estudos que permitem buscar alternativas terapêuticas e sociais, para pessoas que vivem, ou viveram muitos anos em Hospitais Psiquiátricos. Assim, descreveremos a seguir alguns conceitos de reabilitação psicossocial e sua forma de 
ação em saúde mental.

A Reabilitação Psicossocial representa um conjunto de ações, estratégias que são desenvolvidas para beneficiar a vida de pessoas com problemas persistentes em Saúde Mental. Para a Organização Mundial de Saúde, a Reabilitação Psicossocial é o conjunto de atividades capazes de maximizar oportunidades de recuperação de indivíduos e minimizar os efeitos desabilitantes da cronificação das doenças através do desenvolvimento de insumos individuais, familiares e comunitários. Nas sociedades concretas, a brasileira em especial, a pobreza de investimentos na área social irá determinar que alguns recebam cuidados e outros sejam rejeitados pelo sistema de atenção. Serão mais rejeitados os que revelarem uma absoluta inaptidão para o trabalho, já que, no horizonte de expectativas, a inserção no mercado formal ou informal de trabalho entra como indicador positivo em quase todos os projetos de cuidado ${ }^{(2)}$.

Numa tentativa de reintegrar o sofredor psíquico no ambiente social foi criado os CAPS (Centro de Atenção Psicossocial), que é um serviço de saúde aberto e comunitário do Sistema Único de Saúde (SUS). As pessoas atendidas nos CAPS são aquelas que apresentam intenso sofrimento psíquico e que estão impossibilitadas de viver e realizar seus projetos de vida. São, preferencialmente, pessoas com transtornos mentais severos e/ou persistentes, aquelas com grave comprometimento psíquico, incluindo os transtornos relacionados às substâncias psicoativas (álcool e outras drogas) e também crianças e adolescentes com transtornos mentais. Os CAPS visam prestar atendimento em regime de atenção diária, gerenciar os projetos terapêuticos, promover a inserção social dos usuários através de ações intersetoriais e organizar a rede de serviços de saúde mental de seu território e supervisionar a atenção em saúde mental da rede básica $^{(10)}$.

\section{O RELATO DE EXPERIÊNCIA}

Essa experiência foi desenvolvida com oficinas educativas numa Unidade de Hospital Psiquiátrico onde os internos permanecem há muitos anos. Participaram das atividades educativas 16 pessoas, de acordo com avaliação de suas funções psíquicas. Seis delas foram excluídas devido no momento, encontrar-se com seu estado psíquico agravado. Permaneceram então, dez, que foram divididos em dois grupos distintos, cinco com funções psíquicas mais preservadas e cinco que apresentavam algumas alterações significativas, mas que conservavam possibilidade cognitiva, condição mínima necessária para a realização desse trabalho.

\section{As oficinas educativas}

Essas oficinas aconteceram, com o intuito de prestar cuidados de enfermagem através do resgate das habilidades perdidas ao longo do período de internação. As atividades desenvolvidas estavam relacionadas com sua vida diária na unidade, como o cuidado com aparência pessoal, realização de barba, banho, higiene oral, arrumação das camas, organização e cuidados com seus pertences, ingesta da medicação, alimentação e participação em atividades recreativas e laborterápicas.

Essas atividades aconteceram durante quatro semanas no período de agosto a novembro de 2003. Todos os participantes foram informados, na primeira atividade sobre a realização das oficinas e foram convidados voluntariamente a participar do processo. Ressaltamos que seguimos o preconizado pela Resolução 196/96, principalmente os relacionados ao anonimato, voluntariado, acesso às informações coletadas durante os encontros e a liberdade de desistência. Essa atividade fez parte de uma prática assistencial de Trabalho de Conclusão de Curso, sendo avaliada e aprovada por uma banca qualificada de três profissionais da área de Enfermagem Psiquiátrica.

As oficinas aconteceram em etapas explicativas e práticas. Cada uma delas foi realizada uma vez para cada atividade relacionada, durante uma semana; porém, nas duas semanas seguintes, observamos e acompanhamos as atividades desenvolvidas, reorientando, incentivando e ajudando na manutenção das mesmas.

Para tal utilizamos dois momentos: um explicativo e outro prático de desenvolvimento e acompanhamento das atividades. No momento explicativo utilizamos uma técnica de colagem recortando gravuras sobre diversos assuntos relacionados ao cuidado pessoal, colados em papel craft. Nessa atividade, surgiam aspectos sobre o assunto, que eram inicialmente discutidos e relacionados ao cuidado com o corpo. Em seguida nos dirigíamos ao outro local e realizávamos a parte prática da atividade, ou seja, escovação dos dentes, tricotomia facial, entre outras. Num primeiro momento ajudamos individualmente e aos poucos fomos orientando para que eles se conduzissem sozinhos, visando sua 
independência progressiva. As oficinas foram desenvolvidas refletindo sobre a importância de manter uma boa higiene oral, mostrando livros com fotos ilustrativas onde eles tivessem a oportunidade de aprender através da imagem, as partes de um dente saudável, por exemplo.

Cada oficina foi desenvolvida abordando um tema específico relacionada ao cuidado pessoal, a importância de usar talheres (garfo e faca), alimentação saudável, enfim atividades do cotidiano perdidas ao longo dos anos de internação psiquiátrica. O uso da medicação, horários, efeitos, foram abordados, orientando-os da importância na recuperação individual. A ordem com os pertences pessoais, a arrumação dos leitos, a falta de habilidade no desenvolvimento da atividade também foram temas explicativos mostrados como fazer. Por último, enfatizamos a importância da realização de atividades laborativas e recreativas para uma melhor integração social, processo esse que evidencia prejuízos no aumento de seus sintomas.

Essas oficinas mostraram momentos de resgate de habilidades da vida diária perdida com os longos anos de internação, bem como evidenciaram que o portador de transtorno psíquico tem melhores condições de viver. No entanto, é necessário uma atenção próxima, educativa e um acompanhamento tecnicamente adequado das equipes de profissionais de Saúde Mental.

Durante quatro semanas fizemos uma avaliação do estado inicial, por meio de anotações e de observação participante, para estabelecermos uma comparação entre o desenvolvimento das atividades no início do trabalho e no final. Observamos que eles apresentaram diferentes resultados após esse período. Alguns deles, que já desenvolviam algumas das atividades, no final aprimoraram e esclareceram suas dúvidas. Outros que possuíam algumas dificuldades ao término das oficinas apresentavam uma sensível melhora na realização das atividades, reaprendendo a fazê-las. Outros demonstravam interesse em alguns momentos, em outros, desatenção, não progredindo nas atividades, evidenciando o processo de institucionalização no que concerne à perda do interesse e vontade de reaprender novas atividades, talvez devido aos longos anos de internação e pela cronificação dos sintomas progressivos da doença. Alguns deles que apresentaram sintomas delirantes em determinados momentos em outros se mostravam mais integrados, desenvolvendo algumas atividades com maior destreza. Outros permaneceram aparentemente resistentes a todo tipo de novo conhecimento, não parecendo interessados nas atividades. Mesmo assim, no final passaram a realizar pelo menos uma atividade, de higiene corporal, por exemplo, que não desenvolviam há anos, segundo nossas observações e nos relatos da equipe presente da unidade.

Essas atividades desenvolvidas sugerem que, sofredores psíquicos cronificados, quando acompanhados diretamente por profissionais empenhados no processo educativo, respeitados seus limites, fazem progresso considerável das atividades comuns do dia a dia.

Nosso relato de experiência descreve atividades educativas e assistenciais, que foram permeadas por momentos de transformação e aprendizado para todos, sofredores psíquicos e estudantes. Ficou evidenciado que os sofredores psíquicos que participaram das oficinas tornaram-se mais independentes lidando melhor com atividades de vida diária. Essa prática assistencial aprimorou nosso conhecimento, nos permitiu reconhecer a importância de estabelecer vínculos com o sofredor psíquico, evidenciou um resgate da sua auto-estima e conseqüentemente, um melhor viver institucional.

Essa experiência mostrou que uma assistência próxima, afetiva e acolhedora favorece o sofredor psíquico e norteia sua vida mesmo institucionalmente e nos mostra um modelo de prática assistencial comum, presente no nosso dia a dia, todavia carente no ambiente institucional.

\section{ALGUMAS CONSIDERAÇÕES}

Os hospitais psiquiátricos, em sua maioria, ainda mantém uma demanda grande de clientes que estão internados há vários anos na instituição, permanecendo ali, na maioria das vezes, apáticos às intervenções de todos aqueles que trabalham no ambiente hospitalar. Durante a internação, na maior parte do tempo, ficam sem desenvolver nenhuma atividade produtiva e com escassos projetos terapêuticos internos que permitem pouco desenvolvimento de atividades, ou pelo menos, que ocupam pouco espaço do tempo ocioso.

Convivendo durante esse cuidado educativo/ assistencial com os sofredores psíquicos da unidade psiquiátrica em questão, sem uma visão influenciada pelas dificuldades institucionais e sociais, a experiência evidencia que algumas dessas pessoas portadoras de 
sofrimento psíquico, teriam condições de viver em locais de atendimento a pessoas com transtornos psíquicos, em comunidades com recursos especializados, Centro de Atendimento Psicossocial, por exemplo.

Com a realização dessas oficinas, essas pessoas tiveram a oportunidade de desenvolver atividades da vida diária que provavelmente antes da internação desenvolviam e, em consequiência do processo do ócio e da institucionalização, foram perdendo essa habilidade, ao longo dos anos.

Nas instituições psiquiátricas tradicionais, o sofredor psíquico, algumas vezes é considerado incapaz de assumir responsabilidades e de decidir sobre aspectos importantes e simples da própria vida, ficando a instituição e os profissionais que lá atuam responsáveis por suas decisões.

As possibilidades apontadas nesse estudo são viáveis a partir de uma organização dos serviços de saúde mental, principalmente os extra-hospitalares, pois existe pouca oferta de serviços ambulatoriais que asseguram a permanência dos egressos do hospital em outros espaços. A cultura reinante sobre a doença psíquica, o processo de institucionalização prolongado e aspectos vinculados principalmente à deficiente estrutura de serviços extra-hospitalares, limitam de forma especial, os profissionais de saúde mental do hospital, na promoção da reinserção social. Contudo, estamos cientes que possibilidades existem, iniciativas de proporcionar ao sofredor psíquico uma melhor qualidade de vida e, conseqüentemente, seu reingresso na sociedade.

Finalmente, apesar das limitações dessa atividade, principalmente em viabilizar propostas mais efetivas, é evidente que o cuidado de enfermagem associado ao processo educativo é um pressuposto essencial na busca do processo de cidadania perdido e no despertar da consciência para um melhor viver a doença mental. Fica evidente que esse modelo de prática assistencial não deve ficar restrito ao meio acadêmico e nem tampouco a algumas atividades assistenciais isoladas. Todavia, consideramos que ela mostra uma atividade simples na sua ação, mas preciosa no seu resultado. Para desenvolvê-la com qualidade, é necessária habilidade e disposição profissional, além de recursos interinstitucionais, intersetoriais, de capacitação, entre outros, mas acreditamos numa prática acolhedora, humanizada em que se possa atuar com melhor qualidade assistencial.

\section{REFERÊNCIAS}

1. Buchele F, Laurindo PLD, Borges V, Coelho EBS. A interface da saúde mental na atenção básica. Cogitare Enferm. 1996;1(1).

2. Organização Mundial da Saúde. Relatório sobre a saúde no mundo 2001-saúde mental: nova concepção, nova esperança. Geneva: Organização Mundial de Saúde; 2001.

3. Lampert M. Terapia Ocupacional em psiquiatria. Doutor gate: saúde e qualidade de vida [on line]; [citado 15 ago 2003]. Disponível em http://www.drgate.com.br

4. Pitta A. Reabilitação psicossocial no Brasil. São Paulo; Hucitec; 1996.

5. Espinosa AF. Guias práticos de enfermagem: psiquiatria. Rio de Janeiro: Mc Graw-Hill Interamericana do Brasil; 1998.

6. Taylor CM. Fundamentos de enfermagem psiquiátrica. $13^{\mathrm{a}}$ ed. Porto Alegre: Artes Médicas; 1992.

7. Stone MH. A cura da mente: a história da psiquiatria da antiguidade até o presente. Porto Alegre Artmed; 1999.

8. Costa E. Problematizando para humanizar: uma proposta de transformação do cuidado em uma enfermaria psiquiátrica [dissertação]. Florianópolis (SC). Universidade Federal de Santa Catarina; 2002.

9. Buchele F. A enfermagem na reestruturação da assistência psiquiátrica. Texto Contexto Enferm. 1996 Jul/Dez;2(5):140-46.

10. Ministério da Saúde (BR). Secretaria de Atenção à Saúde. Departamento de Ações Programáticas Estratégicas. Saúde mental no SUS: os centros de atenção psicossocial / Ministério da Saúde, Secretaria de Atenção à Saúde, Departamento de Ações Programáticas Estratégicas. Brasília: Ministério da Saúde; 2004. 